\title{
Tarifpolitische Initiativen zum Ausbildungseinstieg und zur Übernahme von Ausgebildeten
}

\author{
THOMAS HAY, WOLFGANG NETTELSTROTH, NORBERT HÜSSON
}

Bis 2050 wird die Bevölkerung in Deutschland laut Schätzungen des Statistischen Bundesamtes um rund 7 Mio. Menschen auf 75 Mio. schrumpfen. Dieser demografische Wandel, sprich die Bevölkerungsentwicklung, hat drei Ursachen: Erstens ist die Sterberate seit Jahrzehnten höher als die Geburtenrate, zweitens steigt die Lebenserwartung, und drittens nimmt der Zuzug durch Migrantinnen und Migranten ab.

Der demografische Wandel verändert die Arbeitswelt erheblich. Das nordrhein-westfälische Arbeitsministerium bezeichnet ihn als „Herausforderung für Unternehmen und Beschäftigte“: Im Jahre 2000 stellte die Gruppe der 50- bis 64-Jährigen noch 30 \% aller Erwerbstätigen. Bereits 2015 werden zwei von fünf Erwerbstätigen älter als 50 Jahre sein. Die 20- bis 34-Jährigen werden dagegen nur noch $30 \%$ der Erwerbstätigen ausmachen. Ein Problem, das daraus für die Unternehmen resultiert, lautet: Es wird schwieriger, junge Beschäftigte zu rekrutieren. Was tun, wenn die Zahl der Schulabgänger sinkt, zugleich die Zahl der Menschen steigt, die altersbedingt ausscheiden, aber der Bedarf an Arbeitskräften bleibt? In Nordrhein-Westfalen (NRW) schrumpft die Zahl der Abgänger in nur sieben Jahren um $20 \%$ : von $540.840 \mathrm{im} \mathrm{Jahr} 2013$ um 111.120 auf $429.720 \mathrm{im} \mathrm{Jahr} \mathrm{2020.}{ }^{\circ}$ Sinkende Schülerzahlen bedeuten: Erst mangelt es an Auszubildenden, dann an Fachkräften. Diesem Problem haben sich als Erste die Tarifvertragsparteien der nordrhein-westfälischen Metall- und Elektroindustrie gestellt: Der Arbeitgeberverband Metall NRW und die IG Metall NRW haben Anfang 2008 den „Tarifvertrag zur Förderung von Ausbildungsfähigkeit“ (TV FAF) geschlossen; er ist seit 1. April 2008 in Kraft.

Angesichts der Sorge um künftige Fachkräftelücken und mit dem Willen, allen Schulabgängern eine gute Ausbildung zu bieten, fanden die Metall-Arbeitgeber und die IG Metall eine neue Antwort auf die vielfach beklagte fehlende oder nur mangelhafte Ausbildungsreife Jugendlicher. Anstatt zu lamentieren, reagierten die Tarifvertragsparteien praktisch: Wenn Jugendliche noch zusätzliche Voraussetzungen für einen erfolgreichen Start in die Ausbildung brauchen, dann müssen sie für diesen Start besonders gefördert werden. Das Motto von Arbeitgeberverband und Gewerkschaft lautet: qualifizieren statt aussortieren. Ausbildungsbetriebe, so ihr Konsens, könnten nicht nur olympiareife Bewerber erwarten; sie müssten sich verstärkt für diejenigen interessieren, die keine Einser-Kandidaten in der Schule waren. Wer dort schlechte Leistungen zeigte oder nur schulmüde war, könne in der Ausbildung trotzdem gut oder hellwach sein. Manche Jugendliche bräuchten eine zweite Chance und mehr Zeit für die Ausbildung statt weniger. Damit gehen die Tarifparteien einen völlig anderen Weg als den der lange umstrittenen Schmalspurausbildung. Denn die Anforderungen in der Ausbildung zu senken, hat bestenfalls zur Folge, dass die Ausgebildeten Billigjobs erhalten; sie haben auf diese Weise zwar die Aussicht auf eine Beschäftigung, aber keine Chancen, dem Niedriglohnsektor zu entfliehen.

\section{Die Eckpunkte des TV FAF}

Der Tarifvertrag zur Förderung der Ausbildungsfähigkeit eröffnet einen neuen Weg in die Berufsausbildung: Wer an der Förderung mit Erfolg teilnimmt, wird anschließend in ein Ausbildungsverhältnis übernommen. Das Ziel ist gleichzeitig der „Hauptgewinn“: ein Ausbildungsplatz.

Die Zielgruppe des TV FAF sind Absolventen der 10. Klasse; es sind Jugendliche, die deshalb keinen Ausbildungsplatz erhalten haben, weil ihre Schulnoten zu schlecht sind und deren Lehrer oder Berufsberater folglich eine Förderung empfehlen.

Die Förderung dauert bis zu einem Jahr. Sie endet unmittelbar vor Beginn des neuen Ausbildungsjahres, „um einen nahtlosen Übergang von der Förderphase in die Ausbildung sicherzustellen“ ( $\$ 3$ Abs. 1 TV FAF). Die Förderung umfasst „Maßnahmen zur Verbesserung der Fachkenntnisse sowie des Arbeits- und Sozialverhaltens“ ( $\$ 3$ Abs. 4 TV FAF). Dazu zählt auch der Einsatz in der Produktion.

Die Teilnehmenden gelten weder als Beschäftigte noch als Auszubildende. Sie haben einen Schülerstatus und mit dem Arbeitgeber einen Fördervertrag geschlossen. Er sieht eine Vergütung vor, die an die Ausbildungsvergütung im ersten Ausbildungsjahr gekoppelt ist: Sie beträgt in

www.arbeit-demografie.nrw.de.

(2) Ministerium für Schule und Weiterbildung des Landes Nordrhein-Westfalen (2012): Schülerprognose und Schulabgängerprognose bis zum Schuljahr 2029/30, Statistische Übersicht (376), Juli, S. 23. 
den ersten drei Monaten der Förderung $75 \%$ der Ausbildungsvergütung, in den folgenden drei Monaten $80 \%$, nach weiteren drei Monaten $85 \%$ und danach bis zum Ende der Förderung $90 \%$. Die Jugendlichen besuchen an zwei Wochentagen das Berufskolleg und arbeiten an drei Tagen im Betrieb. Sie sollen sich Schlüsselqualifikationen aneignen.

Der Betrieb ist „verpflichtet“ ( $\$ 6$ TV FAF), die Teilnehmenden in eine Berufsausbildung zu übernehmen - vorausgesetzt, die Teilnahme war erfolgreich. Die Entscheidung darüber treffen Geschäftsleitung und Betriebsrat gemeinsam. Ein Katalog von Kriterien hilft, die erworbenen Fachkenntnisse sowie das Arbeits- und Sozialverhalten zu beurteilen. Dazu zählen mathematische Grundkenntnisse, die grundlegende Beherrschung der deutschen Sprache sowie Pünktlichkeit, Zuverlässigkeit, Ausdauer und Leistungsbereitschaft, Teamfähigkeit und „Konfliktverhalten“.

\section{Aus dem Dornröschenschlaf erweckt}

Doch trotz der neuen Option, die der TV FAF von Anbeginn eröffnet hat: Der Tarifvertrag war kein Selbstläufer; er erfüllte anfangs die Erwartungen der Tarifparteien nicht, weil er von den Betrieben kaum aufgegriffen wurde. Die Gründe dafür sind nicht eindeutig auszumachen. „Geburtsfehler“ wies das Vertragswerk - auch im Rückblick gesehen - keine auf. Vielleicht unterschätzten die Unternehmen den drohenden Fachkräftemangel oder sie spürten keinen unmittelbaren Handlungsdruck. Vielleicht fehlten auch anfangs geeignete Protagonisten. Letzteres sollte sich zwei Jahre später ändern: Der „Verein zur Förderung der Berufsbildung Dr. Heinz Potthoff“, Bielefeld, startete 2010 eine „Initiative zur Umsetzung des Tarifvertrages FAF - in Kooperation mit Metall-NRW und IG Metall-NRW, unterstützt von Sustain Consult und gefördert mit Geld aus dem Europäischen Sozialfonds (ESF).

Der Verein leistete Pionierarbeit. Zunächst informierte er sich über Erfahrungen mit dem Tarifvertrag: In fünf Unternehmen, die den TV FAF anwandten, fanden Expertengespräche mit Geschäftsleitung und Betriebsrat statt: bei Miele in Gütersloh, Salzgitter Mannesmann Präzisrohr in Hamm, Durable in Kamen, Hille \& Müller in Düsseldorf und Kirchhoff in Olpe und Attendorn. Diese fünf Pilotbetriebe nutzten den TV FAF, entweder, weil sie bereits Schwierigkeiten hatten, geeignete Auszubildende zu finden, oder aus sozialer Verantwortung, weil sie benachteiligten Jugendlichen eine Chance geben wollten. Die „FAFlinge“, so das Kürzel für die nach dem TV FAF in die Förderung einbezogenen Jugendlichen, waren entweder von der Arbeitsagentur vermittelt worden oder hatten sich dank eines Aufrufs des regionalen Unternehmerverbandes gemeldet. Bei der Auswahl legten die Betriebe mehr Wert auf die Kopfnoten der Abschlusszeugnisse als auf die sonstigen Schulnoten. Ein wichtiges Auswahlkriterium war die Einsatzbereitschaft der jungen Leute, die in kurzen Praktika geprüft wurde. Die Jugendlichen wurden in erster Linie für einfache produktive Tätigkeiten eingesetzt, wobei sie hier, wie in der
Ausbildungswerkstatt, vom Ausbildungsleiter betreut wurden.

Mit dem erworbenen Hintergrundwissen informierte der Verein zur Förderung der Ausbildungsfähigkeit die Unternehmen der Metall- und Elektroindustrie in drei Regionen (Siegen/Olpe und Attendorn, Düsseldorf und Bielefeld) über den TV FAF - schriftlich, auf Veranstaltungen und in Einzelgesprächen. Im April 2011 folgte der sogenannte Präsidentenbrief, der Seltenheitswert besitzt und nur zu besonderen Anlässen verschickt wird: Metall-Präsident HorstWerner Maier-Hunke warb für den TV FAF und appellierte an die Unternehmen: „Nutzen auch Sie diese Chance zur eigenen Nachwuchssicherung!" Denn angesichts sinkender Schulabgängerzahlen sei es „,eher unrealistisch“, auf die besseren Bewerber zu hoffen.

Die Vergütung der Jugendlichen entsprechend TV FAF stieß zunähst auf Vorbehalte in den Unternehmen. Der Potthoff-Verein klärte mit der NRW-Regionaldirektion der Agentur für Arbeit, dass der TV FAF und das Bundesprogramm Einstiegsqualifizierung (EQ) - ein betriebliches Langzeitpraktikum - kombiniert werden können. Die örtlichen Agenturen wurden darauf hingewiesen, dass der TV FAF mit EQ-Mitteln gefördert werden kann und soll. Die Agenturen vermittelten auch die Bewerber.

Der Verein zur Förderung der Berufsbildung erreichte in den Pilotregionen 106 Unternehmen und weitere 19 in den Regionen Arnsberg/Hamm, Dortmund, Duisburg, Essen, Gütersloh, Iserlohn/Hagen, Krefeld, Remscheid und Wuppertal. Ein landesweiter Erfahrungsaustausch mit Unternehmen fand im Mai 2011 statt, ein weiterer im Mai 2012. Im Juni 2012 wurde ein Erfahrungsaustausch der Berufskollegs veranstaltet. Dabei stellte sich heraus, dass sich die Beschulung der „FAFlinge“ in den Fachklassen bewährt hat. Ihre Aufnahme in die sogenannten KSOB-Klassen (Klassen für Schülerinnen und Schüler ohne Berufsausbildungsverhältnis) wurde nicht empfohlen.

Bis Ende Juni 2012 boten 28 Unternehmen 53 Ausbildungsvorbereitungsstellen an. 30 Stellen konnten besetzt werden, 23 zunächst nicht, weil die Bewerber entweder nicht die Mindestkriterien erfüllten, also beispielsweise nicht motiviert waren, oder weil keine Bewerber gefunden wurden.

Der Verein zur Förderung der Berufsbildung hat eine Internetseite zum TV FAF geschaltet. ${ }^{3}$ In dem dort verfügbaren Video sagt der Ausbildungsleiter bei Miele: „Wir wissen alle, dass der Fachkräftemangel kommen wird, dass die Schulabgängerzahlen die nächsten Jahre ja sehr stark nach unten zeigen. Und ich glaube, dass über diesen Weg“" - gemeint ist die Ausbildungsvorbereitung nach TV FAF - „eben auch die vermeintlich Schwachen eine Chance haben werden, einen Ausbildungsplatz zu finden.“ Warum, erklärt ein „FAFling“ bei Kirchhoff, so: „Man hat die Möglichkeit, sich zu beweisen, zu zeigen, was man drauf hat,

(3) www.tvfaf.de 
wie man arbeitet [...]" Ein anderer sagt, wenn er die Ausbildungsvorbereitung hinter sich habe, versuche er „natürlich, höher zu kommen - Werkzeugmechaniker oder so."Sein Ausbildungsleiter ist optimistisch. Weil man sich sehr um die Jugendlichen kümmere, „entwickeln sie sich auch sehr gut“, sie hätten das Unternehmen „bis jetzt nicht enttäuscht“.

Diese Erfahrungen können verallgemeinert werden. In einer Befragung der durch den TV FAF Geförderten, an der sich 23 Jugendliche beteiligten, stellte sich heraus:

- Für die Jugendlichen waren drei Gründe ausschlaggebend, den TV FAF zu nutzen: die Chance auf einen Ausbildungsplatz, das Interesse am Ausbildungsplatz, fehlende Alternativen.

- Zwischen Schulabschluss und Beginn der Ausbildungsvorbereitung lagen durchschnittlich drei Jahre, in denen zumeist die Berufsschule besucht, ein Praktikum nach dem anderen absolviert oder gejobbt wurde.

- Die durchschnittliche Dauer der Ausbildungsvorbereitung betrug acht Monate.

- $95 \%$ der Jugendlichen fühlten sich gut oder sehr gut auf die Ausbildung vorbereitet, niemand hat an den Abbruch der Ausbildungsvorbereitung gedacht.

- Alle Jugendlichen empfehlen ihren Freunden den TV FAF.

Relevant ist auch, dass der Bericht des Potthoff-Vereins feststellen kann, dass die Praxis in den Unternehmen zeige, dass der TV FAF keine Ausbildungsplätze ersetzen würde, sondern dass die Unternehmen entweder freie Ausbildungskapazitäten für einen Ausbildungsvorbereitungsplatz nutzten oder zusätzliche Plätze einrichten würden. Ferner seien keine Fälle bekannt, in denen Jugendliche in der Ausbildungsvorbereitungsphase als billige Arbeitskräfte eingesetzt wurden. ${ }^{\circ}$

Alle 30 „FAFlinge“ im Potthoff-Projekt haben einen Ausbildungsplatz erhalten (bis auf einen, der die Ausbildungsvorbereitung abgebrochen hat). Im Abschlussbericht heißt es: „Damit bietet der TV FAF Jugendlichen ohne eine Ausbildungsstelle eine hohe Chance auf Ausbildung und sogar den Einstieg in eine sozialversicherungspflichtige Beschäftigung. “ Der Bericht schlussfolgert, dass der TV FAF sich bewährt habe, in der Unternehmenslandschaft mittlerweile „eingeführt und bekannt“ sei, in den Schulen und Berufskollegs jedoch „noch zu wenig“. Empfohlen wird - neben der Organisation eines Erfahrungsaustauschs der Unternehmen - verbindliche Strukturen zur Zusammenarbeit von Unternehmen und Berufskollegs zu schaffen.

\section{Zu viele Töpfe}

Das ist eine ungewöhnlich positive Bilanz in einer von berufsvorbereitenden Maßnahmen reichen Landschaft: Immerhin gibt es insgesamt 126 Initiativen, Programme und Bildungsgänge allein in NRW. Das hat das Institut für Arbeit und Technik (IAT) der Fachhochschule Gelsenkirchen ermittelt. ${ }^{\bullet}$ Die Vielzahl der Angebote sei „kaum noch überschaubar und vor allem nicht aufeinander abgestimmt", stellt das Forscherteam fest. Zuvor hatte das Arbeitsministerium in Düsseldorf eine noch dramatischere Feststellung getroffen: „Die Ergebnisse und die Kosten-Nutzen-Relation [der berufsvorbereitenden Bildungsangebote, d. Verf.] sind bescheiden - die Akzeptanz sowie der Stellenwert bei den meisten Beteiligten durchgängig eher gering. “ ${ }^{\star 0} \mathrm{Um}$ das zu ändern, ist das Modellvorhaben „Ein-Topf - Berufsvorbereitung aus einer Hand“ ins Leben gerufen worden. ${ }^{\circledR}$ Ziel ist „eine transparente Angebotsstruktur der Berufsvorbereitung“; den Jugendlichen werde „so eine [...] individuelle Förderung bis hin zur Ausbildungsreife ermöglicht. “®

Arbeitsminister Guntram Schneider (SPD) kündigte in einer Pressemitteilung vom 10. September 2012 an: „Wir wollen die jungen Leute frühzeitig bei der Berufsorientierung unterstützen, damit sie nach dem Ende ihrer Schulzeit keine Zeit in Warteschleifen verlieren und schneller und besser in die Berufswelt starten können."

Nordrhein-Westfalen regelt als erstes Flächenland den Übergang von der Schule in den Beruf neu - eine Verabredung mit den Partnern im Ausbildungskonsens. Schneiders Motto: „Kein Abschluss ohne Anschluss.“(1) Zurzeit fehlt über 70.000 jungen Menschen der Anschluss an die Berufswelt - sie stecken in irgendwelchen Maßnahmen.

Mit dem TV FAF haben die IG Metall und der Metallarbeitgeberverband NRW ein Instrument zum erfolgreichen Übergang von Schule in Ausbildung entwickelt, eine echte Alternative zu den zahlreichen Warteschleifen. Denn Kernstück dieses Vertrags ist die Verpflichtung der Unternehmen, den Jugendlichen einen Ausbildungsplatz anzubieten. Die Jugendlichen lernen die Praxis kennen und die Betriebe die Jugendlichen.

(4) Verein zur Förderung der Berufsbildung Dr. Heinz Potthoff e.V. (Hrsg.) (2012): Endbericht: Förderung von Ausbildungsfähigkeit in der Metall- und Elektroindustrie, Bielefeld/Düsseldorf.

(5) Ebd. S. 36.

6 Vgl. IAT (2011): Vom Maßnahme-Dschungel zu mehrTransparenz - Systematisierung der Initiativen, Programme und Bildungsgänge im Übergang von der Schule in den Beruf in NRW, IAT Forschung Aktuell (04).

(7) http://www.arbeit.nrw.de/pdf/ausbildung/rahmenkonzept_ eintopf.pdf.

8 http://www.arbeit.nrw.de/ausbildung/uebergang_schule_ beruf/laufende_massnahmen/ein-topf/index.php.

(9) Ebd.

(10) http://www.arbeit.nrw.de/ausbildung/uebergang_schule_ beruf/index.php.

(11) Ebd. 


\section{Erst Übernahme in Ausbildung, dann in Arbeit}

Damit ist die erste Hürde auf dem Weg ins Berufsleben genommen. Die zweite folgt jedoch drei oder vier Jahre später, nach der Abschlussprüfung: gemeint ist die Übernahme in ein unbefristetes Beschäftigungsverhältnis. Auch dieses Problem hat die IG Metall tarifpolitisch geregelt: im November 2011 in der Stahlindustrie, im Mai 2012 in der Metall- und Elektroindustrie.

Zuvor hatten die Betriebe in der Mehrzahl eine Praxis entwickelt, die Auszubildenden im Regelfall nur befristet zu übernehmen, entsprechend der tariflichen Absicherung, mit der zumindest der Anspruch auf ein volles Arbeitslosengeld erreicht wurde. Das ließ die Ausgebildeten für weitere zwölf bzw. 24 Monate in Unsicherheit. In der Stahlindustrie mussten sich die Auszubildenden bis dato damit zufrieden geben, nur befristet (zuletzt immerhin für 24 Monate) übernommen zu werden. Das änderte sich mit dem Verhandlungsergebnis, das im November 2011 in Düsseldorf erzielt wurde: Seitdem ist die unbefristete Übernahme der Regelfall.

Die unbefristete Übernahme als Grundsatz gilt ab Januar 2013 auch in der Metall- und Elektroindustrie. Die Arbeitgeber und der Betriebsrat können in einer Betriebsvereinbarung festlegen, wie viele der Auszubildenden später unbefristet übernommen werden. Kommt es zu keiner Betriebsvereinbarung, muss der Arbeitgeber sechs Monate vor Ausbildungsende erklären, wie viele Auszubildende er unbefristet übernimmt. Wer demnach über Bedarf ausgebildet wurde, hat Anspruch darauf, für mindestens zwölf Monate übernommen zu werden; drei Monate vor Ablauf dieser Befristung muss der Arbeitgeber prüfen, ob eine Weiterbeschäftigung möglich ist. Der neue Metalltarifvertrag gilt für Auszubildende, deren Ausbildung 2013 und später endet. Der Anspruch auf Übernahme kann mit Zustimmung des Betriebsrates entfallen, wenn die Firma akute Beschäftigungsprobleme hat.

Sowohl für die Stahl- als auch für die Metall- und Elektroindustrie gilt aber, dass Beschäftigungsprobleme nicht per se zu einer Nichtübernahme der Ausgebildeten führen müssen. Rechtfertigt die aktuelle Situation im Betrieb keine Vollzeitbeschäftigung, kann den Ausgebildeten auch eine Beschäftigung in Teilzeit angeboten werden. Selbst die Übernahme in Kurzarbeit oder in einen anderen Betrieb oder in ein anderes Unternehmen ist möglich. Betriebe, die ihre gut ausgebildeten Fachkräfte halten wollen, haben verschiedene Möglichkeiten, gemeinsam kreative und tragfähige Lösungen zu entwickeln.

\section{Fazit}

Mit tarifpolitischen Initiativen ist der Übergang von der Schule in die Ausbildung und von der Ausbildung in den Beruf erfolgreich zu gestalten, für die junge Generation wie für die Unternehmen. Die Tarifpolitik stützt damit das System der dualen Berufsausbildung in Deutschland, dass sich gerade in den letzten Jahren als ein Eckpfeiler industrieller Entwicklungsfähigkeit bewährt hat und deshalb zunehmend in anderen Ländern aufgegriffen wird.

An den skizzierten tarifvertraglichen Regelungen wird deutlich, dass die Berufsausbildung in Deutschland große Integrationskraft besitzt, allemal, wenn das duale System durch Anpassungsmaßnahmen gestützt wird. Die Frühförderung über den TV FAF sorgt dafür, dass mit zusätzlichen Fördermaßnahmen auch die Integration von in der Schule leistungsschwachen Jugendlichen gelingen kann. Ansätze, die auf mehr Förderung in der betrieblichen Praxis setzen, wirken deutlich besser als die praxisfernen „Warteschleifen“ oder die verkürzten, theoriegeminderten Ausbildungsgänge. Die im TV FAF festgeschriebene Verpflichtung, Jugendliche, die die Fördermaßnahme erfolgreich absolviert haben, in eine reguläre Berufsausbildung zu übernehmen, motiviert die Jugendlichen. Sie erhalten damit eine längerfristige Perspektive. Der TV FAF schafft wirksame Übergänge in die Ausbildung für bisher Gescheiterte: mit der gezielten Zugangsförderung und der geregelten Übernahme nach der Ausbildung. Und auch Betriebe, die vom demografisch bedingten Fachkräftemangel bedroht sind, profitieren. Es zeigt sich, der demografische Wandel ist gestaltbar, mit Vorteilen für die Menschen heute wie für unsere künftige wirtschaftliche Leistungsfähigkeit.

\section{AUTOREN}

THOMAS HAY ist IG Metall-Bezirkssekretär in Nordrhein-Westfalen (NRW).

thomas.hay@igmetall.de

WOLFGANG NETTELSTROTH ist IG Metall-Bezirkssekretär in NRW.

wolfgang.nettelstroth@igmetall.de

NORBERT HÜSSON ist freier Journalist in Köln.

norbert.huesson@t-online.de 